\title{
Role of potassium and arbuscular mycorrhizal fungi in alleviation of water stress on Vigna mungo
}

\author{
Navnita Sharma, Kuldeep Yadav, Ashok Aggarwal* \\ Department of Botany, Kurukshetra University, Kurukshetra, Haryana 136119, India \\ *Corresponding author, E-mail: aggarwal_vibha@rediffmail.com
}

\begin{abstract}
A pot culture experiment was carried out under greenhouse conditions to study the effect of potassium fertilizer $\left(70 \mathrm{mg} \mathrm{kg}^{-1}, \mathrm{~K}\right.$, and 100 mg kg-1 K2) and arbuscular mycorrhizal fungi (Glomus mosseae and Acaulospora laevis) alone and in different combinations on water stress amelioration of Vigna mungo during three growth stages. Treatment of the plants with mycorrhizal fungi and potassium improved physiological parameters including chlorophyll concentration, membrane stability, phosphatase activity, leaf protein concentration and mycorrhization over the untreated control regardless of water stress treatments. Imposition of water stress at reproductive stage caused maximum reduction in membrane stability, leaf protein concentration, phosphatase activity and mycorrhization as compared to stress during other stages. Treatment combinations of G. mosseae + A. laevis + K2 and G. mosseae + A. laevis + K1 proved to be the best for promoting physiological and morphological parameters studied both in stressed and unstressed plants. A combination of $G$. mosseae and $\mathrm{K} 2$ was the best among double inoculation treatments while G. mosseae was the best among single treatments of either dose of potassium and A. laevis for stress alleviation and growth amelioration. Imposition of water stress during vegetative stage had the least effect on the physiological parameters studied, except for leaf area, which was adversely affected. Chlorophyll concentration was the most affected parameter by water stress at the pod formation stage.
\end{abstract}

Key words: black gram, drought tolerance, electrolyte leakage, phosphatase activity, legume, mycorrhizal fungi, Vigna mungo. Abbreviations: AL, Acaulospora laevis; AMF, arbuscular mycorrhizal fungi; DAS, days after sowing; GM, Glomus mosseae.

\section{Introduction}

Black gram [Vigna mungo (L.) Hepper], an important food legume, is one of the Asiatic species of pan-tropical genus Vigna. The pulse legume has its origin in the Indian subcontinent, where it has a long history of cultivation as carbonized grains (Vavilov 1926). The seeds have high nutritive value with about $26 \%$ protein, which is thrice that of cereals. Pulse legume is not only important as food and nutritive feed but it also plays a vital role in sustaining soil fertility as well as soil physical properties through symbiotic nitrogen fixation. It is the staple legume of semi-arid and sub-tropical areas of South East Asia. Due to its wider adaptability to semi-arid climates it is an ideal crop for dry land farming. India ranks first in production and consumption of black gram in the world. Over the last decades, there has been an increase in production of $V$. mungo due to better agricultural and breeding techniques but the increase is not enough to meet domestic consumption in the country (Gautam et al. 2016). One of the important reasons for decreased productivity is water stress experienced by the crop during different stages of growth, as nearly $87 \%$ of the area under cultivation of pulses is rain fed.

Under the context of climate change, water deficit is becoming a severe problem restraining plant growth and productivity of terrestrial ecosystems, chiefly in arid regions. It is estimated that water stress can potentially reduce world crop yield by up to $20 \%$ (Scheiermeier 2008). Water is required at every stage of plant growth from seed germination to maturation; any type of imbalance in water acquisition causes a serious threat to agriculture affecting ultimately the yield. Furthermore, the effect of water stress on growth is determined by genotypes, the duration and severity of water deficit, age and stage of plant growth during which stress has occurred (Barnabás et al. 2008).

In order to cope with environmental hazards and struggle against water stress, plants develop various defense approaches; mutualistic association of arbuscular mycorrhizal fungi (AMF) with plant in roots is an example (Wang, Qiu 2006). In this symbiotic association, the intra-radical mycelium propagates in the cortex layer of root and the extraradical mycelium of fungus penetrates through the contact regions of roots in the soil causing better exploration of soil volume for improving uptake of water and mineral nutrients (Wu et al. 2013). Besides this, AMF increase soil aggregate stability by releasing glomalin, a glycoprotein, into the soil (Bedini et al. 2010). Under water deficit conditions, mycorrhizal fungi ameliorate water status of plant by direct water uptake through 
extraradical hyphae, regulation of stomatal conductance, improved phosphorus $(\mathrm{P})$ nutrition due to higher activity of phosphatases, increased photosynthetic pigment concentration and antioxidant enzyme activity, as well as osmotic adjustment by different osmolytes (Sharma et al. 2015). In addition to mycorrhizal fungi, potassium enables the plants to overcome stress by efficient water utilization from soil, increased growth of roots and nutrient uptake. Potassium also regulates the opening of stomata, increases photosynthetic rate and thus yield (Umar 2006).

Considering the wide adaptability and drought tolerance of V. mungo it can be an ideal crop for sustainable agricultural systems in arid regions where crops are stricken by frequent drought stress. For cultivation in such conditions, the drought tolerance of crop needs to be further improved to obtain maximum productivity. To strengthen the cultivation of $V$. mungo in such drought affected areas of Haryana (India), the present study was aimed to investigate whether AMF enhances drought tolerance and to elucidate physiological responses of AMF colonized plants exposed to water stress at different growth stages.

\section{Materials and methods}

\section{Study site and soil characteristics}

The experiment was a laid in a randomized block design with eleven treatments replicated five times, during the kharif season of 2014 and 2015 in a cage house at the Botany Department, Kurukshetra University, Kurukshetra. Top soil used for the experiment consisted of sand (64.2\%), silt $(21.81 \%)$, clay $(3.90 \%)$, with initial $\mathrm{pH} 6.8$, electrical conducticvity $0.25 \mathrm{dS} \mathrm{m}^{-1}$, total nitrogen $0.042 \%$, organic carbon $0.06 \%$, available $\mathrm{K} 0.022 \mathrm{~kg} \mathrm{~m}^{-2}$, available P 0.0018 $\mathrm{kg} \mathrm{m}^{-2}$ and available $\mathrm{S} 14.80 \mathrm{mg} \mathrm{kg}^{-1}$.

\section{Inoculum preparation}

Dominant AMF spores were isolated from the rhizosphere of $V$. mungo by wet sieving and decanting technique of Gerdemann and Nicolson (1963) and identified using the key of Schenck and Perez (1990). Glomus mosseae, also known as Funneliformis mosseae (T. H. Nicolson and Gerd.), C. Walker and A. Schüßler, as well as Acaulospora laevis Gerdemann and Trappe, were found to be the dominant AMF. Starter inoculum of the AMF was raised using the funnel technique of Menge and Timmer (1982). The two AMF species were propagated with maize as a host for a period of three months under pot culture conditions. Bradyrhizobium japonicum culture (procured from Department of Microbiology, CCS Haryana Agricultural University, India) was multiplied by using nutrient broth medium.

\section{Experimental setup}

The experiment was laid in a randomized block design. Five replicates of each treatment were used. Soil and sand in ratio
3:1 was sterilized and added to each earthen pot $(25 \times 25$ $\mathrm{cm})$. Sand was added to increase porosity of the soil. Also, $10 \%$ selected AMF inoculum G mosseae (GM) or A. laevis $(\mathrm{AL})$ with around 860 spores and nearly $80 \%$ colonized root segments of trap host maize was added. Each pot was filled with $1.8 \mathrm{~kg}$ soil sand mixture; $180 \mathrm{~g}$ of inoculum (ie $10 \%$ of the soil mass in the pot) was added for single mycorrhizal inoculation while $90 \mathrm{~g}$ inoculum of each mycorrhizal fungus was added for double inoculation treatment (AL + GM). Potassium was added to the soil after germination in the form of muriate of potash at 70 and $100 \mathrm{mg} \mathrm{kg}^{-1}$ concentration alone and in combinations with mycorrhizal treatments. Seeds of $V$. mungo (procured from Haryana Agriculture University Hisar, Haryana, India) after surface sterilization with $10 \%$ sodium hypochlorite solution were sown in the pots. To each pot, including an uninoculated pot, $10 \mathrm{~mL}$ Bradyrhizobium sp. culture suspension with density $10^{8}$ cells $\mathrm{mL}^{-1}$ was applied. Pots were treated either with single inoculum, combined inoculum or no inoculum as summarized below.

There were four sets of identical treatments as listed above [no water stress, water stress at vegetative stage 25 days after sowing (DAS), reproductive stage 45 DAS and pod formation stage $80 \mathrm{DAS}]$. For every set, each of the eleven treatments was replicated five times. Water stress was applied only once for a particular stage (at vegetative stage 25 DAS, reproductive stage 45 DAS and pod formation stage 80 DAS) for 10 subsequent days (the plants began wilting), then the plants were watered for next 20 days. After 20 days of rewatering, the plants overcame stress and resumed normal growth and were analyzed for different parameters. The set of well watered plants was analysed at vegetative stage. During well watered treatment soil water content was kept at $80 \%$ of the field capacity. During water stress treatment, the soil water was kept at $40 \%$ field capacity by weighing the pots every day to control soil water content.

A preliminary experiment was carried out to fine tune the drought stress period (data not shown). After a 10day period of water stres at $40 \%$ field capacity, the plants

Table 1. Treatments used in the present study

\begin{tabular}{|ll}
\hline No. & Treatments \\
1 & $\begin{array}{l}\text { Control (uninoculated i.e. autoclaved sterile sand/soil } \\
\text { with no inoculum) }\end{array}$ \\
\hline 2 & $\mathrm{~K} 1$ (potassium fertilizer in concentration $70 \mathrm{mg} \mathrm{kg}^{-1}$ ) \\
\hline 3 & $\mathrm{~K} 2$ (potassium fertilizer in concentration $100 \mathrm{mg} \mathrm{kg}^{-1}$ ) \\
\hline 4 & G. mosseae $(\mathrm{GM})$ \\
\hline 5 & A. laevis $(\mathrm{AL})$ \\
\hline 6 & $\mathrm{GM}+\mathrm{K} 1$ \\
\hline 7 & $\mathrm{GM}+\mathrm{K} 2$ \\
\hline 8 & $\mathrm{AL}+\mathrm{K} 1$ \\
\hline 9 & $\mathrm{AL}+\mathrm{K} 2$ \\
\hline 10 & $\mathrm{GM}+\mathrm{AL}+\mathrm{K} 1$ \\
\hline 11 & $\mathrm{GM}+\mathrm{AL}+\mathrm{K} 2$ \\
\hline
\end{tabular}


started wilting; defoliation appeared at the time when they received severer drought (at $30 \%$ of field capacity). Thus, we chose $40 \%$ of field capacity as drought stress for 10 days in order to allow the plants to fully recover after this stress period. Chlorophyll concentration, leaf area, electrolyte leakage, protein concentration of the fresh leaves were determined in the $4^{\text {th }}$ leaf from the growing tip of the plant bythe following methods.

\section{Measurement of physiological and morphological parameters}

Chlorophyll and carotenoid concentration was estimated according to the method of Arnon (1949). Fresh leaf samples $(0.1 \mathrm{~g})$ were homogenised in a pre-chilled pestle and mortar with $80 \%$ acetone under dark conditions. To avoid photodestruction of chlorophyll, a pinch of $\mathrm{CaCO}_{3}$ was added. The suspension was centrifuged at $2000 \mathrm{rpm}$ for $15 \mathrm{~min}$. The supernatant was collected and the volume was made up to $20 \mathrm{~mL}$ with $80 \%$ acetone. The absorbance of the solution was red at $645 \mathrm{~nm}$ and $663 \mathrm{~nm}$ against the solvent (acetone) blank was determined.

Leaf area $\left(\mathrm{cm}^{2}\right)$ was measured by using leaf area meter (Systronics 211, Ahmedabad, India).

The electrolyte leakage test was performed by cutting $200 \mathrm{mg}$ of fresh leaf sample into small discs of $5 \mathrm{~mm}$ diameter. The discs were placed in test tubes containing 10 $\mathrm{mL}$ of deionized water. The tubes were covered with cotton plugs and placed in a water bath at temperature $32 \pm 8^{\circ} \mathrm{C}$. After $2 \mathrm{~h}$ initial electrical conductivity of medium (EC1) was measured using an electrical conductivity meter. The samples were autoclaved at $121 \pm 8^{\circ} \mathrm{C}$ for $20 \mathrm{~min}$ to release all the electrolytes. Samples after cooling were analyzed for final electrical conductivity of medium (EC2). Electrolyte leakage percentage was estimated by using the formula of Dionisio-Sese and Tobita (1998):

Electrolyte leakage $=\mathrm{EC} 1 / \mathrm{EC} 2 \times 100$.

Total protein concentration was estimated by the method of Bradford (1976). In this experiment, $100 \mathrm{mg}$ of the leaf sample was warmed in $10 \mathrm{~mL} 80 \%$ ethanol in a water bath for 2 to $5 \mathrm{~min}$. Then it was cooled at room temperature and homogenized with the same ethanol. The homogenate was centrifuged at $5000 \mathrm{rpm}$ for $10 \mathrm{~min}$. The residue was reextracted with $10 \mathrm{~mL}$ of $5 \%$ perchloric acid with same speed and time. Supernatent was again discarded and the residue was reextracted with $5 \mathrm{~mL}$ of $1 \mathrm{~N} \mathrm{NaOH}$ by keeping it in warm water at temperature 40 to $50^{\circ} \mathrm{C}$ for 30 min. After this, centrifugation was done at $5000 \mathrm{rpm}$ for 10 min and the supernatent was used as protein source.

The protein source taken was $0.2 \mathrm{~mL}$ in each test tube to which $0.8 \mathrm{~mL}$ of double distilled water was added. It was followed by addition of $5 \mathrm{~mL}$ of Comassie Brilliant Blue (protein estimating reagent) to the test tube with immediate shaking at room temperature. Blank was made of $1 \mathrm{~mL}$ double distilled waterwith no plant extract along with $5 \mathrm{~mL}$ of dye. Absorbance was measured at $595 \mathrm{~nm}$ in UV-VIS spectrophotometer (Specord-205, Analytik Jena, Germany). The protein content of the samples was determined against a standard calibration curve of bovine serum albumin (Sigma, USA).

Phosphatase activity was estimated by using $p$-nitrophenyl phosphate as a substrate, which is hydrolyzed by the enzyme to $p$-nitrophenol (Tabatabi, Bremer 1969). Fresh roots were used for extraction of acid and alkaline phosphatases. For this, $1 \mathrm{~g}$ of fresh washed roots were homogenized in $5 \mathrm{~mL}$ of ice cold sodium acetate buffer $(0.1 \mathrm{M}$ with $\mathrm{pH} 4.8)$ for acid phosphatase and sodium carbonate/bicarbonate buffer $(0.05 \mathrm{~N}$ with $\mathrm{pH} 10)$ for alkaline phosphatase activity using a prechilled pestle and mortar. The resulting homogenate was centrifugrd at 10000 rpm for $15 \mathrm{~min}$ and supernatant thus obtained; referred to as crude extract was used for assay of phosphatase activity which was expressed in terms of units per $g$ fresh mass.

\section{Identification and quantification of the number and colonization by AMF spores}

AMF spores were isolated by wet seiving and decanting technique (Gerdemann, Nicolson 1963). Identification of AMF spores (F. mosseae and A. laevis) was done by using the identification manual of Scheneck and Perez (1990); Morton and Benny (1990) and Mukerji (1996). Quantification of AMF spores was done using the Adholeya and Gaur 'Grid Line Intersect Method' (1994). For assesment of mycorrhizal colonization of roots, the roots from each treatment were washed gently with tap water and rinsed with distilled water. A sub sample of 0.5 $\mathrm{g}$ was taken from each root which was then cut into $1 \mathrm{~cm}$ long segments. These segments were bleached for $30 \mathrm{~min}$ in $10 \% \mathrm{KOH}$ at $90{ }^{\circ} \mathrm{C}$ and then were acidified for $10 \mathrm{~min}$ in $1 \% \mathrm{HCl}$. The roots were then stained in trypan blue (Phillips, Hayman 1970) was used to estimate Mycorrhizal colonization of roots. Percent mycorrhizal colonization of roots was calculated by using the formula: (Number of root segments colonized / number of root segments studied) $\times$ 100. One hundred root segments were examined for each particular treatment to determine the root colonization.

\section{Statistical analysis}

Data were subjected to analysis of variance and means separated using the least significant difference test in the Statistical Package for Social Sciences (ver.11.5, Chicago, IL, USA).

\section{Results}

\section{Chlorophyll concentration}

In the present experiment, a reduction in the concentration of photosynthetic pigments in water stressed plants, in contrast to unstressed plants regardless of the various treatments. All the untreated and treated plants in well watered conditions had higher chlorophyll concentration 
Table 2. Effect of AM fungi and potassium on chlorophyll content, leaf area and electrolyte leakage of Vigna mungo grown under well

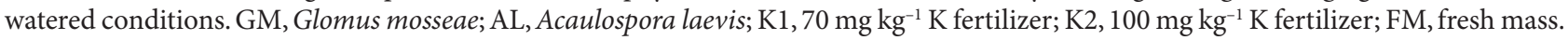
Each value is a mean of five replicates $\pm \mathrm{SD}$. Values in column followed by same letter are not significantly different $(P \leq 0.05)$

\begin{tabular}{|c|c|c|c|c|c|}
\hline \multirow[t]{2}{*}{ Treatment } & \multicolumn{3}{|c|}{ Chlorophyll concentration ( $\left.\mathrm{mg} \mathrm{g}^{-1} \mathrm{FM}\right)$} & \multirow[t]{2}{*}{ Leaf area $\left(\mathrm{cm}^{2}\right)$} & \multirow{2}{*}{$\begin{array}{l}\text { Electrolyte leakage } \\
(\%)\end{array}$} \\
\hline & Chl $a$ & Chl $b$ & Total Chl & & \\
\hline Control & $0.973 \pm 0.003 \mathrm{k}$ & $0.322 \pm 0.003 j$ & $1.296 \pm 0.006 \mathrm{k}$ & $14.10 \pm 2.626 f$ & $28.17 \pm 0.351 \mathrm{a}$ \\
\hline K1 & $1.263 \pm 0.003 \mathrm{j}$ & $0.379 \pm 0.003 \mathrm{i}$ & $1.643 \pm 0.006 \mathrm{j}$ & $17.01 \pm 3.261 \mathrm{f}$ & $27.70 \pm 0.370 \mathrm{~b}$ \\
\hline K2 & $1.327 \pm 0.002 \mathrm{i}$ & $0.395 \pm 0.005 h$ & $1.723 \pm 0.005 \mathrm{i}$ & $21.41 \pm 3.020 \mathrm{e}$ & $27.05 \pm 0.388 \mathrm{c}$ \\
\hline GM & $1.734 \pm 0.002 \mathrm{~g}$ & $0.530 \pm 0.004 f$ & $2.265 \pm 0.004 \mathrm{~g}$ & $26.60 \pm 3.106 \mathrm{~d}$ & $26.13 \pm 0.306 \mathrm{e}$ \\
\hline $\mathrm{AL}$ & $1.533 \pm 0.004 h$ & $0.451 \pm 0.004 \mathrm{~g}$ & $1.984 \pm 0.007 \mathrm{~h}$ & $22.15 \pm 2.569 \mathrm{e}$ & $26.55 \pm 0.370 \mathrm{~d}$ \\
\hline $\mathrm{GM}+\mathrm{K} 1$ & $2.133 \pm 0.016 \mathrm{~d}$ & $0.703 \pm 0.002 \mathrm{~d}$ & $2.837 \pm 0.016 \mathrm{~d}$ & $31.41 \pm 2.964 \mathrm{c}$ & $25.14 \pm 0.288 \mathrm{~g}$ \\
\hline $\mathrm{GM}+\mathrm{K} 2$ & $2.615 \pm 0.003 b$ & $1.040 \pm 0.003 b$ & $3.656 \pm 0.004 b$ & $34.12 \pm 1.880 c$ & $24.20 \pm 0.140 \mathrm{i}$ \\
\hline $\mathrm{AL}+\mathrm{K} 1$ & $1.746 \pm 0.003 \mathrm{f}$ & $0.531 \pm 0.004 f$ & $2.278 \pm 0.006 f$ & $26.27 \pm 2.328 \mathrm{~d}$ & $25.53 \pm 0.243 f$ \\
\hline $\mathrm{AL}+\mathrm{K} 2$ & $2.028 \pm 0.002 \mathrm{e}$ & $0.628 \pm 0.003 e$ & $2.657 \pm 0.005 e$ & $26.20 \pm 5.813 \mathrm{~d}$ & $24.70 \pm 0.167 \mathrm{~h}$ \\
\hline $\mathrm{GM}+\mathrm{AL}+\mathrm{K} 1$ & $2.496 \pm 0.003 c$ & $1.001 \pm 0.003 c$ & $3.498 \pm 0.005 c$ & $48.61 \pm 1.639 \mathrm{a}$ & $23.53 \pm 0.118 j$ \\
\hline $\mathrm{GM}+\mathrm{AL}+\mathrm{K} 2$ & $2.684 \pm 0.002 a$ & $1.155 \pm 0.003 a$ & $3.840 \pm 0.006 a$ & $44.28 \pm 1.976 b$ & $21.93 \pm 0.149 \mathrm{k}$ \\
\hline L.S.D $(P \leq 0.05)$ & 0.0073 & 0.0051 & 0.0095 & 3.8632 & 0.3575 \\
\hline ANOVA F $_{(10,23)}$ & 502.04 & 266.44 & 664.34 & 61.900 & 222.869 \\
\hline
\end{tabular}

than that in stress conditions (Table 2 to 5). Among the stressed and unstressed plants, well-watered plants with triple inoculation with GM $+\mathrm{AL}+\mathrm{K} 2$ had the highest chlorophyll $a$ concentration followed by GM + K2 (Table 2 ). The same treatments had the highest chlorophyll a concentration in plants stressed at reproductive as well as pod formation stages (Table 4 and 5). The plants exposed to stress during vegetative stage had maximum chlorophyll $a$ concentration when treated with GM $+\mathrm{AL}+$ $\mathrm{K} 2$ followed by GM $+\mathrm{AL}+\mathrm{K} 1$ (Table 2). Treatment GM + $\mathrm{AL}+\mathrm{K} 2$ was the best in respect to chlorophyll $b$ and total chlorophyll concentration for all stressed and unstressed plants. However, plants stressed during vegetative stage had comparatively higher chlorophyll concentration than those stressed during reproductive stage (Table 3 and 4), but treatments $\mathrm{GM}+\mathrm{K} 2$ and $\mathrm{AL}+\mathrm{K} 1$ resulted in higher chlorophyll concentration in the plants stressed at reproductive stage than those stressed during vegetative stage.

\section{Leafarea}

Among treatments, maximum leaf area was recorded in treatment $\mathrm{GM}+\mathrm{AL}+\mathrm{K} 1$ under well watered conditions (Table 2). When stress was given at vegetative stage, maximum leaf area occurred in treatment GM and $\mathrm{GM}+\mathrm{K} 2$ (Table 3). G. mosseae when used alone and in combination with potassium resulted in higher leaf area than in the A. laevis treatment. At reproductive stage,

Table 3. Effect of AM fungi and potassium on chlorophyll concentration, leaf area and electrolyte leakage of Vigna mungo grown under

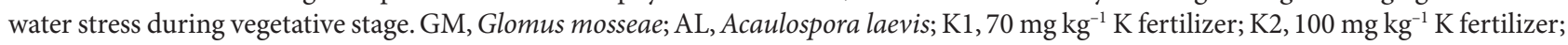
$\mathrm{FM}$, fresh mass. Each value is a mean of five replicates \pm SD. Values in column followed by same letter are not significantly different $(P$ $\leq 0.05)$

\begin{tabular}{|c|c|c|c|c|c|}
\hline \multirow[t]{2}{*}{ Treatment } & \multicolumn{3}{|c|}{ Chlorophyll concentration ( $\left.\mathrm{mg} \mathrm{g}^{-1} \mathrm{FM}\right)$} & \multirow[t]{2}{*}{ Leaf area $\left(\mathrm{cm}^{2}\right)$} & \multirow{2}{*}{$\begin{array}{c}\text { Electrolyte leakage } \\
(\%)\end{array}$} \\
\hline & Chl $a$ & Chl $b$ & Total Chl & & \\
\hline Control & $0.765 \pm 0.002 \mathrm{k}$ & $0.214 \pm 0.004 j$ & $0.981 \pm 0.006 \mathrm{k}$ & $6.54 \pm 1.80 \mathrm{i}$ & $32.72 \pm 0.177 \mathrm{a}$ \\
\hline K1 & $1.150 \pm 0.003 \mathrm{j}$ & $0.319 \pm 0.005 i$ & $1.469 \pm 0.008 \mathrm{j}$ & $9.25 \pm 2.25 \mathrm{~h}$ & $30.01 \pm 0.453 b$ \\
\hline $\mathrm{K} 2$ & $1.302 \pm 0.003 \mathrm{i}$ & $0.379 \pm 0.003 h$ & $1.682 \pm 0.006 \mathrm{i}$ & $12.11 \pm 1.56 \mathrm{~g}$ & $28.63 \pm 0.236 c$ \\
\hline GM & $1.531 \pm 0.002 \mathrm{~g}$ & $0.490 \pm 0.004 \mathrm{f}$ & $2.022 \pm 0.007 \mathrm{~g}$ & $39.09 \pm 2.41 \mathrm{a}$ & $26.71 \pm 0.343 e$ \\
\hline $\mathrm{AL}$ & $1.387 \pm 0.005 h$ & $0.418 \pm 0.004 \mathrm{~g}$ & $1.805 \pm 0.006 h$ & $13.78 \pm 1.64 \mathrm{fg}$ & $27.41 \pm 0.119 \mathrm{~d}$ \\
\hline $\mathrm{GM}+\mathrm{K} 1$ & $2.127 \pm 0.002 \mathrm{~d}$ & $0.690 \pm 0.005 \mathrm{~d}$ & $2.817 \pm 0.007 \mathrm{~d}$ & $21.12 \pm 2.69 \mathrm{de}$ & $25.84 \pm 0.148 f$ \\
\hline $\mathrm{GM}+\mathrm{K} 2$ & $2.393 \pm 0.004 c$ & $0.876 \pm 0.003 c$ & $3.269 \pm 0.006 c$ & $36.19 \pm 2.01 b$ & $24.30 \pm 0.168 \mathrm{~g}$ \\
\hline $\mathrm{AL}+\mathrm{K} 1$ & $1.618 \pm 0.014 f$ & $0.488 \pm 0.007 f$ & $2.089 \pm 0.047 f$ & $18.60 \pm 1.95 \mathrm{e}$ & $26.09 \pm 0.332 f$ \\
\hline $\mathrm{AL}+\mathrm{K} 2$ & $2.004 \pm 0.002 \mathrm{e}$ & $0.593 \pm 0.009 \mathrm{e}$ & $2.602 \pm 0.007 e$ & $21.61 \pm 2.08 \mathrm{~cd}$ & $24.31 \pm 0.247 \mathrm{~g}$ \\
\hline $\mathrm{GM}+\mathrm{AL}+\mathrm{K} 1$ & $2.538 \pm 0.003 b$ & $0.964 \pm 0.004 b$ & $3.503 \pm 0.005 b$ & $23.97 \pm 1.62 c$ & $23.50 \pm 0.139 h$ \\
\hline $\mathrm{GM}+\mathrm{AL}+\mathrm{K} 2$ & $2.614 \pm 0.002 a$ & $1.043 \pm 0.004 a$ & $3.658 \pm 0.005 a$ & $15.44 \pm 1.96 f$ & $22.94 \pm 0.171 \mathrm{i}$ \\
\hline L.S.D $(P \leq 0.05)$ & 0.0066 & 0.0065 & 0.0202 & 2.5878 & 0.3408 \\
\hline ANOVA F $_{11}$ & 690.94 & 144.14 & 154.64 & 128.942 & 631.272 \\
\hline
\end{tabular}


Table 4. Effect of AM fungi and potassium on chlorophyll content, leaf area and electrolyte leakage of Vigna mungo grown under water stress during reproductive stage. GM, Glomus mosseae; AL, Acaulospora laevis; K1, $70 \mathrm{mg} \mathrm{kg}^{-1} \mathrm{~K}_{\text {fertilizer; }} \mathrm{K} 2,100 \mathrm{mg} \mathrm{kg}$ ' $\mathrm{K}_{\text {fertilizer; }}$ FM, fresh mass. Each value is a mean of five replicates \pm SD. Values in column followed by same letter are not significantly different $(P$ $\leq 0.05)$

\begin{tabular}{|c|c|c|c|c|c|}
\hline \multirow[t]{2}{*}{ Treatment } & \multicolumn{3}{|c|}{ Chlorophyll content ( $\left.\mathrm{mg} \mathrm{g}^{-1} \mathrm{FM}\right)$} & \multirow[t]{2}{*}{ Leaf area $\left(\mathrm{cm}^{2}\right)$} & \multirow{2}{*}{$\begin{array}{c}\text { Electrolyte leakage } \\
(\%)\end{array}$} \\
\hline & Chl $a$ & Chl $b$ & Total Chl & & \\
\hline Control & $0.699 \pm 0.003 \mathrm{k}$ & $0.166 \pm 0.005 \mathrm{i}$ & $0.865 \pm 0.004 \mathrm{k}$ & $8.92 \pm 1.412 \mathrm{~h}$ & $45.03 \pm 0.820 \mathrm{a}$ \\
\hline $\mathrm{K} 1$ & $1.118 \pm 0.003 \mathrm{j}$ & $0.283 \pm 0.009 \mathrm{~h}$ & $1.402 \pm 0.011 \mathrm{j}$ & $11.79 \pm 2.056 \mathrm{~g}$ & $38.26 \pm 0.366 b$ \\
\hline K2 & $1.228 \pm 0.007 i$ & $0.359 \pm 0.014 \mathrm{~g}$ & $1.586 \pm 0.012 \mathrm{i}$ & $14.32 \pm 1.711 \mathrm{fg}$ & $44.04 \pm 0.689 \mathrm{a}$ \\
\hline GM & $1.492 \pm 0.006 \mathrm{~g}$ & $0.430 \pm 0.008 \mathrm{f}$ & $1.923 \pm 0.006 \mathrm{~g}$ & $18.52 \pm 1.752 \mathrm{de}$ & $33.10 \pm 0.260 \mathrm{~d}$ \\
\hline $\mathrm{AL}$ & $1.362 \pm 0.002 \mathrm{~h}$ & $0.357 \pm 0.007 \mathrm{~g}$ & $1.720 \pm 0.010 \mathrm{~h}$ & $16.41 \pm 1.547 \mathrm{ef}$ & $35.06 \pm 0.112 c$ \\
\hline $\mathrm{GM}+\mathrm{K} 1$ & $1.891 \pm 0.002 \mathrm{~d}$ & $0.444 \pm 0.007 \mathrm{e}$ & $2.336 \pm 0.009 \mathrm{~d}$ & $22.87 \pm 1.972 c$ & $29.91 \pm 0.232 \mathrm{e}$ \\
\hline $\mathrm{GM}+\mathrm{K} 2$ & $2.400 \pm 0.003 b$ & $0.724 \pm 0.006 c$ & $3.126 \pm 0.005 c$ & $40.61 \pm 2.044 \mathrm{a}$ & $28.26 \pm 0.210 f$ \\
\hline $\mathrm{AL}+\mathrm{K} 1$ & $1.661 \pm 0.003 f$ & $0.430 \pm 0.008 \mathrm{f}$ & $2.092 \pm 0.005 f$ & $20.55 \pm 2.284 c d$ & $33.70 \pm 0.374 \mathrm{~d}$ \\
\hline $\mathrm{AL}+\mathrm{K} 2$ & $1.718 \pm 0.001 \mathrm{e}$ & $0.461 \pm 0.009 \mathrm{~d}$ & $2.181 \pm 0.009 \mathrm{e}$ & $22.50 \pm 3.243 c$ & $33.16 \pm 0.207 d$ \\
\hline $\mathrm{GM}+\mathrm{AL}+\mathrm{K} 1$ & $2.366 \pm 0.002 c$ & $0.789 \pm 0.007 b$ & $3.151 \pm 0.010 \mathrm{~b}$ & $28.24 \pm 1.639 b$ & $24.80 \pm 0.662 \mathrm{~g}$ \\
\hline $\mathrm{GM}+\mathrm{AL}+\mathrm{K} 2$ & $2.494 \pm 0.004 a$ & $0.898 \pm 0.005 a$ & $3.413 \pm 0.041 \mathrm{a}$ & $38.84 \pm 1.917 \mathrm{a}$ & $25.31 \pm 0.478 g$ \\
\hline L.S.D $(P \leq 0.05)$ & 0.0042 & 0.011 & 0.0194 & 2.5721 & 1.0164 \\
\hline $\operatorname{ANOVA~F}_{(10,23)}$ & 156.05 & 333.83 & 137.24 & 128.844 & 378.176 \\
\hline
\end{tabular}

imposition of water stress had less adverse effect on leaf area of the plants, compared to those stressed during vegetative stage; treatment of plants with $\mathrm{GM}+\mathrm{AL}+\mathrm{K} 1$ and $\mathrm{GM}+$ $\mathrm{AL}+\mathrm{K} 2$ resulted in maximum leaf area (Table 4). Among the three stages, plants subjected to water stress at pod formation stage had largest leaf area (Table 5). Water stress at pod formation stage caused the least effect on the leaf area because, during this stage, plant leaves could expand to the maximum as no water stress was imposed.

\section{Electrolyte leakage}

In the present experiment, maximum rate of cell membrane damage (as electrolyte leakage) was recorded when the plants were stressed during reproductive stage in untreated control plants. Treatment of plants with $\mathrm{GM}+\mathrm{AL}+\mathrm{K} 1$ at this stage resulted in minimum membrane damage (Table 4). Of the three stages of stress imposition, highest membrane stability was observed in plants treated with $\mathrm{GM}+\mathrm{AL}+\mathrm{K} 2$ during vegetative stage (Table 3). Among stressed and unstressed plants, maximum stability of membrane was reported in well watered plants with the same treatment (Table 2). Combination of mycorrhizal fungi with potassium was quite effective as compared to single inoculation treatment with either of the AMF used in the experiment.

Table 5. Effect of AM fungi and potassium on chlorophyll content, leaf area and electrolyte leakage of Vigna mungo grown under water stress during pod formation stage. GM, Glomus mosseae; AL, Acaulospora laevis; $\mathrm{K} 1,70 \mathrm{mg} \mathrm{kg}{ }^{-1} \mathrm{~K}_{\text {fertilizer; }} 2$, $100 \mathrm{mg} \mathrm{kg}{ }^{-1} \mathrm{~K}$ fertilizer; FM, fresh mass. Each value is a mean of five replicates \pm SD. Values in column followed by same letter are not significantly different $(P$ $\leq 0.05)$

\begin{tabular}{|c|c|c|c|c|c|}
\hline \multirow[t]{2}{*}{ Treatment } & \multicolumn{3}{|c|}{ Chlorophyll content ( $\left.\mathrm{mg} \mathrm{g}^{-1} \mathrm{FM}\right)$} & \multirow[t]{2}{*}{ Leaf area $\left(\mathrm{cm}^{2}\right)$} & \multirow{2}{*}{$\begin{array}{c}\text { Electrolyte leakage } \\
(\%)\end{array}$} \\
\hline & Chl $a$ & Chl $b$ & Total Chl & & \\
\hline Control & $0.551 \pm 0.003 \mathrm{k}$ & $0.163 \pm 0.016 \mathrm{k}$ & $0.818 \pm 0.023 j$ & $10.37 \pm 1.909 \mathrm{~h}$ & $37.37 \pm 0.040 \mathrm{a}$ \\
\hline K1 & $0.698 \pm 0.003 \mathrm{i}$ & $0.209 \pm 0.004 j$ & $0.906 \pm 0.008 \mathrm{~h}$ & $14.85 \pm 2.112 \mathrm{~g}$ & $34.38 \pm 0.018 b$ \\
\hline K2 & $0.785 \pm 0.002 \mathrm{~h}$ & $0.301 \pm 0.015 h$ & $1.086 \pm 0.013 \mathrm{~g}$ & $18.68 \pm 1.993 \mathrm{f}$ & $33.23 \pm 0.278 c$ \\
\hline GM & $1.056 \pm 0.002 \mathrm{~g}$ & $0.285 \pm 0.005 \mathrm{i}$ & $1.341 \pm 0.007 f$ & $21.47 \pm 3.012 \mathrm{ef}$ & $31.59 \pm 0.403 f$ \\
\hline $\mathrm{AL}$ & $0.649 \pm 0.031 j$ & $0.313 \pm 0.003 g$ & $0.865 \pm 0.006 \mathrm{i}$ & $19.60 \pm 1.732 \mathrm{f}$ & $32.69 \pm 0.193 d$ \\
\hline $\mathrm{GM}+\mathrm{K} 1$ & $1.706 \pm 0.003 \mathrm{~d}$ & $0.512 \pm 0.004 \mathrm{~d}$ & $2.219 \pm 0.007 \mathrm{c}$ & $23.25 \pm 2.514 \mathrm{e}$ & $28.27 \pm 0.153 h$ \\
\hline $\mathrm{GM}+\mathrm{K} 2$ & $1.995 \pm 0.003 b$ & $0.633 \pm 0.003 b$ & $2.552 \pm 0.060 \mathrm{~b}$ & $30.91 \pm 2.401 c$ & $27.78 \pm 0.138 \mathrm{i}$ \\
\hline $\mathrm{AL}+\mathrm{K} 1$ & $1.172 \pm 0.017 f$ & $0.326 \pm 0.002 \mathrm{f}$ & $1.499 \pm 0.017 \mathrm{e}$ & $22.69 \pm 1.774 \mathrm{e}$ & $32.12 \pm 0.199 \mathrm{e}$ \\
\hline $\mathrm{AL}+\mathrm{K} 2$ & $1.539 \pm 0.003 e$ & $0.441 \pm 0.003 \mathrm{e}$ & $1.981 \pm 0.007 \mathrm{~d}$ & $26.34 \pm 1.549 d$ & $30.02 \pm 0.194 \mathrm{~g}$ \\
\hline $\mathrm{GM}+\mathrm{AL}+\mathrm{K} 1$ & $1.931 \pm 0.002 \mathrm{c}$ & $0.556 \pm 0.002 c$ & $2.563 \pm 0.007 b$ & $46.67 \pm 1.879 \mathrm{a}$ & $24.63 \pm 0.703 \mathrm{k}$ \\
\hline $\mathrm{GM}+\mathrm{AL}+\mathrm{K} 2$ & $2.015 \pm 0.004 a$ & $0.747 \pm 0.005 a$ & $2.762 \pm 0.007 \mathrm{a}$ & $40.85 \pm 1.753 b$ & $25.87 \pm 0.074 j$ \\
\hline L.S.D $(P \leq 0.05)$ & 0.0145 & 0.0100 & 0.0149 & 2.6737 & 0.2784 \\
\hline ANOVA F $_{11}$ & 127.44 & 274.73 & 206.34 & 132.327 & 151.83 \\
\hline
\end{tabular}


Table 6. Effect of AM fungi and potassium on some biochemical parameters and mycorrhization of Vigna mungo grown under well

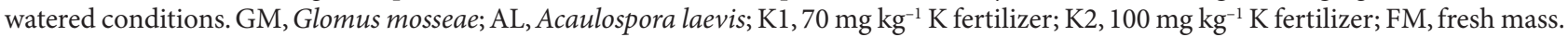
Each value is a mean of five replicates \pm SD. Values in column followed by same letter are not significantly different $(P \leq 0.05)$

\begin{tabular}{|c|c|c|c|c|c|}
\hline \multirow[t]{2}{*}{ Treatment } & \multicolumn{2}{|c|}{ Phosphatase activity (IU g g ${ }^{-1}$ FM) } & \multirow{2}{*}{$\begin{array}{l}\text { AMF spore number } \\
\text { per } 10 \mathrm{~g} \text { of soil }\end{array}$} & \multirow{2}{*}{$\begin{array}{c}\text { AMF root } \\
\text { colonization }(\%)\end{array}$} & \multirow{2}{*}{$\begin{array}{c}\text { Protein } \\
\text { concentration } \\
\left(\mathrm{mg} \mathrm{g}^{-1} \mathrm{FM}\right)\end{array}$} \\
\hline & Acidic & Alkaline & & & \\
\hline Control & $0.069 \pm 0.005 f$ & $0.087 \pm 0.006 h$ & $8.4 \pm 4.393 h$ & $0.4 \pm 0.288 h$ & $0.205 \pm 0.002 j$ \\
\hline K1 & $0.096 \pm 0.006 \mathrm{e}$ & $0.135 \pm 0.005 \mathrm{~g}$ & $24.4 \pm 3.209 g$ & $14.2 \pm 3.193 g$ & $0.194 \pm 0.003 \mathrm{j}$ \\
\hline K2 & $0.101 \pm 0.011 \mathrm{e}$ & $0.141 \pm 0.005 \mathrm{fg}$ & $29.6 \pm 3.847 f$ & $22.8 \pm 4.147 f$ & $0.292 \pm 0.003 \mathrm{i}$ \\
\hline GM & $0.141 \pm 0.005 b$ & $0.186 \pm 0.007 \mathrm{c}$ & $76.0 \pm 3.674 \mathrm{~d}$ & $65.4 \pm 3.714 \mathrm{~d}$ & $0.415 \pm 0.002 \mathrm{~g}$ \\
\hline $\mathrm{AL}$ & $0.111 \pm 0.004 \mathrm{~d}$ & $0.145 \pm 0.008 \mathrm{ef}$ & $69.0 \pm 4.472 \mathrm{e}$ & $53.4 \pm 3.847 \mathrm{e}$ & $0.345 \pm 0.003 h$ \\
\hline $\mathrm{GM}+\mathrm{K} 1$ & $0.125 \pm 0.004 c$ & $0.149 \pm 0.007 \mathrm{ef}$ & $88.6 \pm 4.615 \mathrm{bc}$ & $76.6 \pm 4.277 \mathrm{~b}$ & $0.627 \pm 0.004 \mathrm{~d}$ \\
\hline $\mathrm{GM}+\mathrm{K} 2$ & $0.139 \pm 0.009 b$ & $0.178 \pm 0.005 \mathrm{~d}$ & $92.6 \pm 3.974 \mathrm{ab}$ & $83.8 \pm 4.024 a$ & $0.815 \pm 0.005 a$ \\
\hline $\mathrm{AL}+\mathrm{K} 1$ & $0.113 \pm 0.007 \mathrm{~d}$ & $0.147 \pm 0.006 \mathrm{ef}$ & $83.6 \pm 4.615 c$ & $71.0 \pm 4.301 \mathrm{c}$ & $0.465 \pm 0.002 \mathrm{f}$ \\
\hline $\mathrm{AL}+\mathrm{K} 2$ & $0.132 \pm 0.004 b c$ & $0.152 \pm 0.008 \mathrm{e}$ & $85.6 \pm 3.435 c$ & $73.6 \pm 4.615 b c$ & $0.550 \pm 0.028 \mathrm{e}$ \\
\hline $\mathrm{GM}+\mathrm{AL}+\mathrm{K} 1$ & $0.151 \pm 0.008 \mathrm{a}$ & $0.214 \pm 0.009 \mathrm{~b}$ & $94.8 \pm 3.114 \mathrm{a}$ & $85.6 \pm 3.209 a$ & $0.739 \pm 0.003 c$ \\
\hline $\mathrm{GM}+\mathrm{AL}+\mathrm{K} 2$ & $0.160 \pm 0.005 a$ & $0.239 \pm 0.004 a$ & $94.8 \pm 3.563 a$ & $88.0 \pm 4.301 \mathrm{a}$ & $0.781 \pm 0.002 b$ \\
\hline L.S.D $(P \leq 0.05)$ & 0.009 & 0.008 & 5.0182 & 4.8488 & 0.0228 \\
\hline ANOVA F $_{(10,23)}$ & 71.650 & 193.910 & 322.960 & 332.777 & 317.63 \\
\hline
\end{tabular}

\section{Protein concentration}

Among the stressed plants, minimum protein content was found in plants subjected to water stress at reproductive stage and the treatments $\mathrm{GM}+\mathrm{AL}+\mathrm{K} 2$ followed by GM + $\mathrm{AL}+\mathrm{K} 1$ resulted in maximum protein content in the plants stressed during reproductive and pod formation stage (Table 8 and 9). The treatment GM $+\mathrm{AL}+\mathrm{K} 2$ followed by $\mathrm{GM}+\mathrm{K} 2$ proved to be better treatment for increasing leaf protein concentration in well watered plants and in plants stressed during vegetative stage (Table 6 and 7).

\section{Phosphatase activity}

Imposition of water stress during reproductive stage caused maximum reduction in acid and alkaline phosphatase enzyme activity (Table 8). However, stress during vegetative stage had the least effect on root phosphatase activity (Table 7). Alkaline phosphatase activity was found to be larger than acidic phosphatase activity, and the treatment of plants with $\mathrm{GM}+\mathrm{AL}+\mathrm{K} 2$ and $\mathrm{GM}+\mathrm{AL}+\mathrm{K} 1$ resulted in the highest phosphatase activity (both acid and alkaline) in plants stressed during pod formation stage along with well watered plants (Table 6 and 9), while the plants stressed during reproductive and vegetative stage had maximum phosphatase activity in treatments with $\mathrm{GM}+\mathrm{AL}+\mathrm{K} 2$ and $\mathrm{GM}+\mathrm{K} 2$ (Table 7 and 8 ).

Table 7. Effect of AM fungi and potassium on some biochemical parameters and mycorrhization of Vigna mungo grown under water stress during vegetative stage. GM, Glomus mosseae; AL, Acaulospora laevis; K1, $70 \mathrm{mg} \mathrm{kg}^{-1} \mathrm{~K}$ fertilizer; $\mathrm{K} 2,100 \mathrm{mg} \mathrm{kg}{ }^{-1} \mathrm{~K}$ fertilizer; FM, fresh mass. Each value is a mean of five replicates \pm SD. Values in column followed by same letter are not significantly different $(P \leq 0.05)$

\begin{tabular}{|c|c|c|c|c|c|}
\hline \multirow[t]{2}{*}{ Treatment } & \multicolumn{2}{|c|}{ Phosphatase activity (IU g $\left.{ }^{-1} \mathrm{FM}\right)$} & \multirow{2}{*}{$\begin{array}{l}\text { AMF spore number } \\
\text { per } 10 \mathrm{~g} \text { of soil }\end{array}$} & \multirow{2}{*}{$\begin{array}{c}\text { AMF root } \\
\text { colonization (\%) }\end{array}$} & \multirow{2}{*}{$\begin{array}{c}\text { Protein } \\
\text { concentration } \\
\left(\mathrm{mg} \mathrm{g}^{-1} \mathrm{FM}\right)\end{array}$} \\
\hline & Acidic & Alkaline & & & \\
\hline Control & $0.622 \pm 0.009 g$ & $0.083 \pm 0.007 \mathrm{~g}$ & $0.0 \pm 0.000 \mathrm{~g}$ & $0.0 \pm 0.000 \mathrm{~g}$ & $0.193 \pm 0.003 \mathrm{k}$ \\
\hline K1 & $0.089 \pm 0.005 f$ & $0.128 \pm 0.004 f$ & $0.0 \pm 0.000 \mathrm{~g}$ & $0.0 \pm 0.000 \mathrm{~g}$ & $0.200 \pm 0.004 \mathrm{j}$ \\
\hline K2 & $0.094 \pm 0.005 f$ & $0.135 \pm 0.007 \mathrm{ef}$ & $0.0 \pm 0.000 \mathrm{~g}$ & $0.0 \pm 0.000 \mathrm{~g}$ & $0.255 \pm 0.002 \mathrm{i}$ \\
\hline GM & $0.107 \pm 0.004 \mathrm{e}$ & $0.143 \pm 0.005 \mathrm{~cd}$ & $71.6 \pm 4.393 \mathrm{e}$ & $55.6 \pm 4.277 \mathrm{e}$ & $0.334 \pm 0.003 g$ \\
\hline $\mathrm{AL}$ & $0.104 \pm 0.007 \mathrm{e}$ & $0.139 \pm 0.005 \mathrm{de}$ & $54.8 \pm 3.492 \mathrm{f}$ & $46.0 \pm 4.062 \mathrm{f}$ & $0.295 \pm 0.002 h$ \\
\hline $\mathrm{GM}+\mathrm{K} 1$ & $0.128 \pm 0.009 \mathrm{~cd}$ & $0.150 \pm 0.007 \mathrm{c}$ & $83.6 \pm 4.393 \mathrm{~cd}$ & $71.6 \pm 3.049 \mathrm{c}$ & $0.507 \pm 0.003 \mathrm{~d}$ \\
\hline $\mathrm{GM}+\mathrm{K} 2$ & $0.150 \pm 0.006 \mathrm{ab}$ & $0.234 \pm 0.006 \mathrm{a}$ & $87.0 \pm 3.535 b c$ & $75.2 \pm 3.701 b c$ & $0.717 \pm 0.002 \mathrm{a}$ \\
\hline $\mathrm{AL}+\mathrm{K} 1$ & $0.121 \pm 0.005 \mathrm{~d}$ & $0.146 \pm 0.006 \mathrm{~cd}$ & $74.8 \pm 3.701 \mathrm{e}$ & $66.4 \pm 4.929 \mathrm{~d}$ & $0.417 \pm 0.002 \mathrm{f}$ \\
\hline $\mathrm{AL}+\mathrm{K} 2$ & $0.142 \pm 0.005 b$ & $0.175 \pm 0.006 b$ & $81.6 \pm 4.615 d$ & $71.6 \pm 5.319 c$ & $0.474 \pm 0.002 \mathrm{e}$ \\
\hline $\mathrm{GM}+\mathrm{AL}+\mathrm{K} 1$ & $0.133 \pm 0.003 c$ & $0.172 \pm 0.005 b$ & $90.8 \pm 4.147 \mathrm{ab}$ & $81.4 \pm 4.037 \mathrm{a}$ & $0.602 \pm 0.001 c$ \\
\hline $\mathrm{GM}+\mathrm{AL}+\mathrm{K} 2$ & $0.154 \pm 0.003 a$ & $0.235 \pm 0.003 a$ & $93.0 \pm 3.872 \mathrm{a}$ & $79.2 \pm 3.492 \mathrm{ab}$ & $0.672 \pm 0.002 b$ \\
\hline L.S.D $(P \leq 0.05)$ & 0.0081 & 0.0080 & 4.3903 & 4.5294 & 0.0039 \\
\hline ANOVA F $_{(10,23)}$ & 94.584 & 253.262 & 628.962 & 444.366 & 184.14 \\
\hline
\end{tabular}


Table 8. Effect of AM fungi and potassium on some biochemical parameters and mycorrhization of Vigna mungo grown under water stress during reproductive stage. GM, Glomus mosseae; AL, Acaulospora laevis; K1, $70 \mathrm{mg} \mathrm{kg}^{-1} \mathrm{~K}_{\text {fertilizer; }} \mathrm{K} 2,100 \mathrm{mg} \mathrm{kg}$ ' $\mathrm{K}_{\text {fertilizer; }}$ FM, fresh mass. Each value is a mean of five replicates \pm SD. Values in column followed by same letter are not significantly different $(P$ $\leq 0.05)$

\begin{tabular}{|c|c|c|c|c|c|}
\hline \multirow[t]{2}{*}{ Treatment } & \multicolumn{2}{|c|}{ Phosphatase activity (IU g $\left.{ }^{-1} \mathrm{FM}\right)$} & \multirow{2}{*}{$\begin{array}{c}\text { AMF spore number } \\
\text { per } 10 \mathrm{~g} \text { of soil }\end{array}$} & \multirow{2}{*}{$\begin{array}{c}\text { AMF root } \\
\text { colonization }(\%)\end{array}$} & \multirow{2}{*}{$\begin{array}{c}\text { Protein } \\
\text { concentration } \\
\left(\mathrm{mg} \mathrm{g}^{-1} \mathrm{FM}\right)\end{array}$} \\
\hline & Acidic & Alkaline & & & \\
\hline Control & $0.048 \pm 0.009 f$ & $0.064 \pm 0.005 \mathrm{~g}$ & $0.0 \pm 0.000 \mathrm{~h}$ & $0.0 \pm 0.000 \mathrm{~h}$ & $0.175 \pm 0.003 \mathrm{k}$ \\
\hline K1 & $0.064 \pm 0.004 \mathrm{e}$ & $0.107 \pm 0.004 \mathrm{f}$ & $0.0 \pm 0.000 \mathrm{~h}$ & $0.0 \pm 0.000 \mathrm{~h}$ & $0.184 \pm 0.003 \mathrm{j}$ \\
\hline K2 & $0.071 \pm 0.005 \mathrm{e}$ & $0.120 \pm 0.005 \mathrm{e}$ & $0.0 \pm 0.000 \mathrm{~h}$ & $0.0 \pm 0.000 \mathrm{~h}$ & $0.190 \pm 0.002 \mathrm{i}$ \\
\hline GM & $0.100 \pm 0.011 \mathrm{~cd}$ & $0.129 \pm 0.006 \mathrm{~d}$ & $52.8 \pm 4.147 f$ & $37.0 \pm 3.162 f$ & $0.265 \pm 0.002 \mathrm{~g}$ \\
\hline $\mathrm{AL}$ & $0.092 \pm 0.005 \mathrm{~d}$ & $0.126 \pm 0.008 \mathrm{de}$ & $48.0 \pm 3.674 \mathrm{~g}$ & $29.4 \pm 3.361 \mathrm{~g}$ & $0.209 \pm 0.004 h$ \\
\hline $\mathrm{GM}+\mathrm{K} 1$ & $0.117 \pm 0.006 b$ & $0.150 \pm 0.010 \mathrm{~b}$ & $65.2 \pm 4.549 \mathrm{~cd}$ & $61.2 \pm 3.033 c$ & $0.395 \pm 0.003 \mathrm{~d}$ \\
\hline $\mathrm{GM}+\mathrm{K} 2$ & $0.143 \pm 0.006 a$ & $0.156 \pm 0.005 \mathrm{ab}$ & $69.0 \pm 3.535 c$ & $67.8 \pm 3.420 \mathrm{~b}$ & $0.464 \pm 0.003 c$ \\
\hline $\mathrm{AL}+\mathrm{K} 1$ & $0.107 \pm 0.004 \mathrm{c}$ & $0.133 \pm 0.004 \mathrm{~cd}$ & $59.6 \pm 4.277 \mathrm{e}$ & $43.0 \pm 3.674 \mathrm{e}$ & $0.300 \pm 0.002 \mathrm{f}$ \\
\hline $\mathrm{AL}+\mathrm{K} 2$ & $0.118 \pm 0.005 b$ & $0.138 \pm 0.005 c$ & $63.8 \pm 4.324 \mathrm{de}$ & $55.6 \pm 3.209 \mathrm{~d}$ & $0.345 \pm 0.003 e$ \\
\hline $\mathrm{GM}+\mathrm{AL}+\mathrm{K} 1$ & $0.126 \pm 0.006 b$ & $0.157 \pm 0.006 \mathrm{ab}$ & $74.8 \pm 3.492 b$ & $67.6 \pm 0.714 b$ & $0.507 \pm 0.002 b$ \\
\hline $\mathrm{GM}+\mathrm{AL}+\mathrm{K} 2$ & $0.148 \pm 0.008 a$ & $0.160 \pm 0.006 a$ & $84.6 \pm 4.615 a$ & $74.6 \pm 4.037 a$ & $0.524 \pm 0.002 \mathrm{a}$ \\
\hline L.S.D $(P \leq 0.05)$ & 0.0089 & 0.0081 & 4.4554 & 3.7675 & 0.0037 \\
\hline $\operatorname{ANOVA~F}_{(10,23)}$ & 105.460 & 96.605 & 413.499 & 478.094 & 104.14 \\
\hline
\end{tabular}

\section{Mycorrhization}

Among the three stress stages, imposition of water stress during reproductive stage most affected mycorrhization (Table 8 ). The vegetative stage was least affected by drought stress followed by pod formation stage (Table 7 and 9). During reproductive stage, a large amount of carbon resources is allocated to flowering, and as a result availability of photosynthates to the mycorrhizal symbiont is lower which might have resulted in decreased colonization at this stage. Among the different treatments used, maximum spore number and root colonization were observed on triple inoculation of plants with $\mathrm{GM}+\mathrm{AL}+\mathrm{K} 2$ and $\mathrm{GM}$ $+\mathrm{AL}+\mathrm{K} 1$ indicating stimulatory role of potassium in enhancement of mycorrhization. Mycorrhizal colonization increased when both species were supplemented with potassium in concentration $100 \mathrm{mg} \mathrm{kg}^{-1}$.

\section{Discussion}

In this experiment, decrease in photosynthetic pigments due to water stress could be attributed to drought induced deterioration of thylakoid membranes causing substantial

Table 9. Effect of AM fungi and potassium on some biochemical parameters and mycorrhization of Vigna mungo grown under water

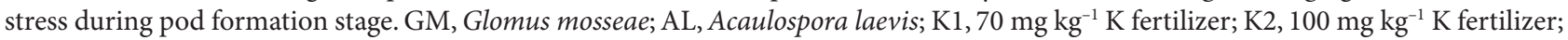
FM, fresh mass. Each value is a mean of five replicates \pm SD. Values in column followed by same letter are not significantly different $(P$ $\leq 0.05)$

\begin{tabular}{|c|c|c|c|c|c|}
\hline \multirow[t]{2}{*}{ Treatment } & \multicolumn{2}{|c|}{ Phosphatase activity (IU g ${ }^{-1}$ FM) } & \multirow{2}{*}{$\begin{array}{l}\text { AMF spore number } \\
\text { per } 10 \mathrm{~g} \text { of soil }\end{array}$} & \multirow{2}{*}{$\begin{array}{c}\text { AMF root } \\
\text { colonization (\%) }\end{array}$} & \multirow{2}{*}{$\begin{array}{c}\text { Protein } \\
\text { concentration } \\
\left(\mathrm{mg} \mathrm{g}^{-1} \mathrm{FM}\right)\end{array}$} \\
\hline & Acidic & Alkaline & & & \\
\hline Control & $0.064 \pm 0.012 \mathrm{~g}$ & $0.079 \pm 0.005 \mathrm{~g}$ & $0.0 \pm 0.000 \mathrm{~g}$ & $0.0 \pm 0.000 \mathrm{f}$ & $0.181 \pm 0.002 \mathrm{k}$ \\
\hline K1 & $0.083 \pm 0.011 \mathrm{f}$ & $0.124 \pm 0.005 f$ & $0.0 \pm 0.000 \mathrm{~g}$ & $0.0 \pm 0.000 \mathrm{f}$ & $0.189 \pm 0.002 \mathrm{j}$ \\
\hline K2 & $0.078 \pm 0.022 \mathrm{f}$ & $0.129 \pm 0.007 \mathrm{ef}$ & $0.0 \pm 0.000 \mathrm{~g}$ & $0.0 \pm 0.000 \mathrm{f}$ & $0.215 \pm 0.003 \mathrm{i}$ \\
\hline GM & $0.102 \pm 0.008 \mathrm{de}$ & $0.135 \pm 0.006 \mathrm{de}$ & $68.2 \pm 3.898 \mathrm{e}$ & $44.0 \pm 4.358 \mathrm{~d}$ & $0.306 \pm 0.003 \mathrm{~g}$ \\
\hline $\mathrm{AL}$ & $0.097 \pm 0.010 \mathrm{e}$ & $0.130 \pm 0.003 \mathrm{def}$ & $49.6 \pm 2.701 \mathrm{f}$ & $34.6 \pm 3.911 \mathrm{e}$ & $0.264 \pm 0.004 h$ \\
\hline $\mathrm{GM}+\mathrm{K} 1$ & $0.123 \pm 0.005 b c$ & $0.167 \pm 0.007 \mathrm{~b}$ & $77.2 \pm 4.147 \mathrm{c}$ & $67.4 \pm 4.827 \mathrm{~b}$ & $0.427 \pm 0.003 \mathrm{e}$ \\
\hline $\mathrm{GM}+\mathrm{K} 2$ & $0.133 \pm 0.004 b$ & $0.167 \pm 0.007 b$ & $83.2 \pm 4.764 b$ & $73.6 \pm 3.361 \mathrm{a}$ & $0.525 \pm 0.007 c$ \\
\hline $\mathrm{AL}+\mathrm{K} 1$ & $0.111 \pm 0.005 \mathrm{~cd}$ & $0.138 \pm 0.005 d$ & $69.6 \pm 4.449 \mathrm{de}$ & $56.4 \pm 3.974 \mathrm{c}$ & $0.386 \pm 0.003 \mathrm{f}$ \\
\hline $\mathrm{AL}+\mathrm{K} 2$ & $0.122 \pm 0.005 b c$ & $0.145 \pm 0.004 c$ & $73.4 \pm 4.037 \mathrm{~cd}$ & $65.6 \pm 3.847 b$ & $0.475 \pm 0.002 d$ \\
\hline $\mathrm{GM}+\mathrm{AL}+\mathrm{K} 1$ & $0.147 \pm 0.005 \mathrm{a}$ & $0.191 \pm 0.006 \mathrm{a}$ & $87.6 \pm 3.847 \mathrm{a}$ & $76.8 \pm 4.919 a$ & $0.585 \pm 0.006 b$ \\
\hline $\mathrm{GM}+\mathrm{AL}+\mathrm{K} 2$ & $0.149 \pm 0.006 a$ & $0.196 \pm 0.004 a$ & $91.2 \pm 3.701 \mathrm{a}$ & $74.2 \pm 3.701 a$ & $0.611 \pm 0.002 \mathrm{a}$ \\
\hline L.S.D $(P \leq 0.05)$ & 0.0131 & 0.0076 & 4.331 & 4.5049 & 0.0036 \\
\hline $\operatorname{ANOVA~F}_{(10,23)}$ & 37.148 & 155.896 & 584.175 & 397.151 & 15870.294 \\
\hline
\end{tabular}


damage to photosynthetic pigments (Anjum et al. 2011). Decreased chlorophyll concentration could be due to higher activities of chlorophyllase causing increased breakdown of chlorophyll (Kaewsuksaeng 2011). Reduction in photosynthetic pigments due to water stress has also been confirmed by Mafakheri et al. (2010) and Din et al. (2011). Mycorrhizal treatment to the plants along with potassium proved beneficial for increasing chlorophyll concentration in the plants despite of water stress treatments. An increase in photosynthetic pigment concentration as a result of mycorrhizal inoculation under drought stressed conditions has been confirmed by Asrar and Elhindi (2011) in Tagetus erecta. The minimum chlorophyll concentration observed in the plants stressed during pod formation stage, among the three stress stages, was because at later growth stages, chlorophyll content decreases due to increasing senescence and the source sink relationship, as observed by Nursu'aidah et al. (2014). In the present experiment, water stress affected leaf area of the plants, and well watered plants had greater leaf area than stressed plants. Reduction in leaf area under water stressed conditions is a mechanism for minimizing higher transpiration rates. Among the three stages of water stress imposition, minimum leaf area has been reported in the plants stressed at vegetative stage. Water stress during vegetative stage caused much greater reduction in leaf area than in the other two stress stages, due to reduction in cell division, and leaf expansion to maximum attainable size. Our results agree with the findings of Mohamed et al. (2010). Mycorrhizal treatment increased leaf area of plants regardless of water stress. Similar results have been noted by Asrar and Elhindi (2011) in mycorrhizal Tagetus erecta. Mycorrhizal fungi increase potassium nutrition of the plant (Gracia, Zimmermann 2014). Dual treatment of AMF fungi and potassium increased leaf area to the maximum because of increased potassium nutrition to the plants, as potassium plays an important role in increasing leaf area of the plants under water stress conditions (Parvez et al. 2006). Increased photosynthetic pigment concentration and leaf area as a result of inoculation with mycorrhizae and potassium may increase photosynthesis, because more light energy will be absorbed by chlorophyll molecules in plant leaves having large surface area to drive photosynthesis (Sheng et al. 2008) in plants. Increase in photosynthesis thus results in enhancement in the yield under stressfull conditions (Parry et al. 2011).

In plant cells, electrolyte leakage is mainly associated with efflux of potassium ions, which is intervened by plasma membrane cation conductances (Demidchik et al. 2014). Potassium nutrition to plants reduces water stress induced membrane damage by deeper penetration of roots, improving root architecture and water retention by the plant tissues (Wang et al. 2013). Compared to the plants stressed at vegetative stage, relatively more membrane damage due to water stress was observed in the plants stressed during reproductive stage. These findings of our study are in agreement with those of Farjam et al. (2014). Mycorrhizal treatment to the water stressed and well watered plants decreased electrolyte leakage, which has also been confirmed by Fouad et al. (2014). Water stress had undesirable effect on leaf protein concentration regardless of potassium and AMF treatments. Well watered plants had maximum leaf protein concentration. Reduction in plant leaf protein concentration during water deficiency may be due to proteolysis or non availability of some vital minerals like nitrogen required for protein synthesis, which are taken up by plants with water. In the present experiment, drought stress affected the concentration of photosynthetic pigments, which might have resulted in decreased photosynthesis by the plants. This reduction in photosynthesis could have affected the availability of compounds required for protein synthesis (Karimi et al. 2012). Similar results have been reported in maize by Abdelmoneim et al. (2013). Increase in leaf protein concentration on mycorrhizal inoculation has also been confirmed (Abdelmoneim et al. 2013). Plants performed better under mycorrhizal treatment with potassium than under mycorrhizal fungi alone, as potassium also improves water relations of drought stressed plants, which increases uptake of essential minerals required for protein formation. Phosphatase enzymes are found in the vacuoles of AMF hyphae, which aid in phosphorus metabolism of plants. They help in mineralization of organic $\mathrm{P}$ into useful forms such as ortho- and polyphosphates, which can be easily utilized by plants (Richardson et al. 2009). In the present experiment, the activity of acid and alkaline phosphatase was weak in uninoculated plants, which increased significantly with mycorrhization.

Combination of arbuscular mycorrhizal fungi and potassium was also found to be helpful in increasing phosphatase activity over that in single treatment of AMF. Higher phosphatase activity has been recorded in mycorrhizal plants by Kumar et al. (2008) and Wu et al. (2011) under water stressed conditions. Role of potassium in improving phosphatase activity has been investigated by Ali and Meihy (2015). Increased phosphatase activity in mycorrhizal plants under drought stress helps in increasing tolerance of plants to water stress by increasing the availability of phosphorus (Stancheva et al. 2008). Increased photosynthetic pigments and leaf area help in increasing photosynthesis membrane stability; phosphorus uptake of mycorrhizal plants increases growth and productivity under water stress conditions. Mycorrhizal root colonization was found to be positively related with leaf area, protein concentration, membrane stability and phosphatase activity, suggesting reduction in electrolyte leakage, improvement in phosphorus metabolism and growth of mycorrhizal plants under well watered and water stressed conditions. Water stress affected mycorrhization, as number of AMF spores and percentage of root colonization in well watered plants was found to be higher than in stressed plants (Table 
5). The reason for this may be slow spread of fungal hyphae after germination of AMF spores due to water deficit. Our results agree with the findings of Gong et al. (2014), who observed inhibition in AMF colonization in foxtail millet roots as a result of water stress, which could be attributed to reduced availability of carbon to the stressed plants (Subramanian, Charest 1995). Our results confirm the findings of Kadian et al. (2013). As different AMF species have different distribution of hyphae in the soil, hyphal density of AMF mycelium surrounding the roots due to colonization by two species will be greater than for single species. Moreover, increase in mycorrhization by two AMF species might be attributed to decreased competition for the form of carbon required by individual AMF species, as the fungi may require different forms of carbon. Crop productivity is affected by stress induced physiological changes. The results of the present investigation suggest the amelioration of physiological parameters of $V$. mungo under water stress conditions on application of G. mosseae, A. laevis and $\mathrm{K} 2$ i.e. $100 \mathrm{mg} \mathrm{kg}^{-1}$ during all the stages of growth.

In conclusion, results of the present experiment could be employed to avoid the wasteful irrigation of crops during any phase of growth. For cultivation of V. mungo, reproductive and pod formation stages were found to be most sensitive to water stress. Thus, irrigation of fields at these two stages may help in obtaining high yield, comparable to when irrigating agricultural land throughout the growing season, which causes inefficient utilization of the natural resource. Water stress during vegetative stage caused minimum damage to the plants while stress during reproductive stage caused maximum damage to the plants in terms of photosynthetic pigments, membrane stability, leaf protein concentration and mycorrhization. G. mosseae proved to be a better bioinoculant as compared to A. laevis, when used in combination or alone, indicating biological specificity of host for a particular microbial strain.

\section{Acknowledgements}

The authors are thankful to Kurukshetra University, Kurukshetra for providing laboratory and infrastructural facilities to carry out the research work.

\section{References}

Abdelmoneim T.S., Moussa T.A.A., Almaghrabi O.A., Hassan S.A., Abdelbagi I. 2013. Increasing plant tolerance to drought stress by inoculation with arbuscular mycorrhizal fungi. Life Sci. J. 10: 255-263.

Adholeya A., Gaur A. 1994. Estimation of VAM fungal spores in soil. Mycorrhiza News 6: 10-11.

Ali M.M.S., Meihy R.E. 2015. Microbiological indicators of a clayey soil planted with wheat (Triticum aestivum L.) as affected by potassium fertilization and different water regimes. Res. J. Soil Biol. 7: 72-83.

Anjum S.A., Xie X. L., Wang M.F., Saleem C.M., Lei W. 2011.
Morphological physiological and biochemical responses of plants to drought stress. Afr. J. Agric. Res. 6: 2026-2032.

Arnon D.I. 1949. Copper enzymes in isolated chloroplasts, polyphenoxidase in Beta vulgaris. Plant Physiol. 24: 1-15.

Asrar A.W.A., Elhindi K.M. 2011. Alleviation of drought stress of marigold (Tagetes erecta) plants by using arbuscular mycorrhizal fungi. Saudi J. Biol. Sci. 18: 93-98.

Barnabás B., Jäger K., Fehér A. 2008. The effect of drought and heat stress on reproductive processes in cereals. Plant Cell Environ. 31: 11-38.

Bedini S., Pellegrino E., Avio L., Pellegrini S., Bazzoffi P., Argese E., Giovannetti M. 2010. Changes in soil aggregation and glomalin-related soil protein content as affected by arbuscular mycorrhizal fungal species Glomus mosseae and Glomus intraradices. Soil Biol. Biochem. 41: 1491-1496.

Bradford M.M. 1976. A rapid and sensitive for the quantization of microgram quantitites of protein utilizing the principle of protein-dye binding. Anal. Biochem. 72: 248-254.

Demidchik V., Straltsova D., Medvedev S.S., Pozhvanov G.A., Sokolik A., Yurin V. 2014. Stress-induced electrolyte leakage: the role of $\mathrm{K}^{+}$-permeable channels and involvement in programmed cell death and metabolic adjustment. J. Exp. Bot. 65: 1259-1270.

Din J., Khan U., Ali I., Gurmani R.A. 2011. Physiological and agronomic response of canola varieties to drought stress. J. Anim. Plant Sci. 21: 78-82.

Dionisio-Sese M.L., Tobita S. 1998. Antioxidant responses of rice seedlings to salinity stress. Plant Sci. 135: 1-9.

Emam Y., Shekoofa A., Salehi F., Jalali A.H. 2010. Water stress effects on two common bean cultivars with contrasting growth habits. Am. Eurasian J. Agric. Environ. Sci. 9: 495-499.

Farjam S., Siosemardeh A., Kazemi-Arbat H., Yarnia M., Rokhzadi A. 2014. Response of chickpea (Cicer arietinum L.) to exogenous salicylic acid and ascorbic acid under vegetative and reproductive drought stress conditions. J. Appl. Bot. Food Qual. 87: 80-86.

Fouad M. O., Essahibi A., Benhiba L., Qaddoury A. 2014. Effectiveness of arbuscular mycorrhizal fungi in the protection of olive plants against oxidative stress induced by drought. Spanish J. Agric. Res. 12: 763-771.

Gautam N.K., Kumar K., Prasad M. 2016. Leaf crinkle disease in urdbean (Vigna mungo L. Hepper): An overview on causal agent, vector and host. Protoplasma 253: 729-746.

Gerdemann J.W., Nicolson Y.H. 1963. Spores of mycorrhizae endogone species extracted from soil by wet sieving and decanting. Transact. Brit. Mycol. Soc. 46: 235-244.

Girigowda K., Prashanth S. J., Mulimani V. H. 2005. Oligosaccharides of black gram (Vigna mungo.L.) as affected by processing methods. Plant Food Human Nutr. 60: 173-180.

Gong M., You X., Zhang Q. 2014. Effects of Glomus intraradices on growth and reactive oxygen metabolism of foxtail millet under drought. Ann. Microbiol. 65: 595-602.

Kadian N., Yadav K., Aggarwal A. 2013. Significance of bioinoculants in promoting growth, nutrient uptake and yield of Cyamopsis tetragonoloba (L.) “Taub." Eur. J. Soil Biol. 58: 66-72.

Kaewsuksaeng S. 2011. Chlorophyll degradation in horticultural crops. Walailak J. Sci. Techn. 8: 9-19.

Karimi S., Abbaspour H., Sinaki J.M., Makarian H. 2012. Effects of water deficit and chitosan spraying on osmotic adjustment and soluble protein of cultivars castor bean (Ricinus communis L.). J. Stress Physiol. Biochem. 8: 160-169. 
Kumar A., Bernier J., Verulkar S., Lafitte H.R., Atlin G.N. 2008. Breeding for drought tolerance: direct selection for yield, response to selection and use of drought-tolerant donors in upland and lowland-adapted populations. Field Crops Res. 107: 221-231.

Mafakheri A., Siosemardeh A., Bahramnejad B., Straik P.C., Sohrabi E. 2010. Effect of drought stress on yield, proline and chlorophyll contents in three chickpea cultivars. Afr. J. Crop Sci. 4: 580-585.

Menge J.A., Timmer L.W. 1982. Procedure for inoculation of plants with VAM in the laboratory, greenhouse and field. In: Schenck N.C. (ed) Methods and Principles of Mycorrhizal Research. American Phytopathology Society, St Paul, pp. 59-67.

Morton J.B., Benny G.L. 1990. Revised classification of arbuscular mycorrhizal fungi (Zygomycetes): A new order, Glomales, two new suborders, Glomineae and Gigasporineae, with an remendation of Glomaceae. Mycotaxon 37: 471-491.

Mukerji K.G. 1996. Taxonomy of endomycorrhizal fungi. In: Mathur K.G., Chamola B., Chitralekha P. (eds) Advances in Botany. APH Pubishing Company, New Delhi, pp. 211-221.

Nursu'aidah H., Motior M.R., Nazia A.M., Islam M.A. 2014. Growth and photosynthetic responses of long bean (Vigna unguiculata) and mung bean (Vigna radiata) response to fertilization. J. Anim. Plant Sci. 24: 573-578.

Phillips J.M., Hayman D.S. 1970. Improved procedures for clearing roots and staining parasitic and VAM fungi for rapid assessment of infection. Transact. Brit. Mycol. Soc. 55: 158-161.

Richardson A.E., Barea J.M., McNeill A.M., Prigent-Combaret C. 2009. Acquisition of phosphorus and nitrogen in the rhizosphere and plant growth promotion by microorganism. Plant Soil 321: 305-339.

Sakova L.R., Paclik Curn V. 2002. The drought tolerance of four Brassica species. Sbornik Jihoceska Univerzita Zemedelska Fakulta Ceske Budejovice Fytotechnicka Rada 1:77-86.

Schenck N.C., Perez Y. 1990. Manual for the Identification of VA Mycorrhizal Fungi. University of Florida Press, Florida, USA.

Schiermeier Q. 2008. Water: a long dry summer. Nature 452: 270273.

Sharma N., Yadav K., Cheema J., Badda N., Aggarwal A. 2015. Arbuscular mycorrhizal symbiosis and water stress: a critical review. Pertanika J. Trop. Agric. Sci. 38: 427-453.
Stancheva M., Geneva E., Djonova N., Kaloyanova M., Sichanova M., Boychinova G., Geogiev. 2008. Response of alfalfa (Medicago sativa L.) growth at low accessible phosphorous source to the dual inoculation with mycorrhizal fungi and nitrogen fixing bacteria. Gen. Appl. Plant Physiol. 34: 319-326.

Subramanian K.S., Charest C. 1995. Influence of arbuscular mycorrhizae on the metabolism of maize under drought stress. Mycorrhiza 5: 273-278.

Tabatabai M. A., Bremner J. M. 1969. Use of p-nitrophenyl phosphate for assay of soil phosphatase activity. Soil Biol. Biochem. 1: 301-307.

Thalooth A.T., Tawfik M.M., Magda Mohamed H. 2006. A comparative study on the effect of foliar application of zinc, potassium and magnesium on growth, yield and some chemical constituents of mung bean plants grown under water stress conditions. World J. Agric. Sci. 2: 37-46.

Umar S. 2006. Alleviating adverse effects of water stress on yield of sorghum, mustard and groundnut by potassium application. Pak. J. Bot. 38: 1373-1380.

Vavilov N. I. 1926. Studies on the origin of cultivated plants. Bulletin of Applied Botany and Plant Breeding 14: 1-245. /in Rusian/

Wang B., Qiu Y.L. 2006. Phylogenetic distribution and evolution of mycorrhizas in land plants. Mycorrhiza 16: 299-363.

Wang M., Zheng Q., Shen Q., Guo S. 2013. The critical role of potassium in plant stress response. Int. J. Mol. Sci. 14: 73707390.

Wu Q.S., Srivastava A.K., Zoua Y. N. 2013. AMF-induced tolerance to drought stress in citrus: A review. Sci. Hort. 164: 77-87.

Wu Q.S., Zou Y.N., He X.H. 2011. Difference of hyphal and soil phosphatase activities in drought-stressed mycorrhizal trifoliate orange (Poncirus trifoliata) seedlings. Sci. Hort. 129: 294-298.

Garcia K., Zimmermann S.D. 2014. The role of mycorrhizal associations in plant potassium nutrition. Front. Plant Sci. 5: 337.

Pervez H.M., Makhdum I., Ashraf M., Shabab-Ud-Din 2006. Influence of potassium nutrition on leaf area index in cotton (Gossypium hirsutum L.) under an arid environment. Pak. J. Bot. 38: 1085-1092. 